\title{
Unresolved Classical Electromagnetic Aspects of the Aharonov-Bohm Phase Shift
}

\author{
Timothy H. Boyer \\ Department of Physics, City College of the City \\ University of New York, New York, New York 10031
}

\begin{abstract}
The long-standing controversy regarding the Aharonov-Bohm phase shift is reviewed. The shifts of both optical and particle interference patterns are summarized. It is pointed out that a line of electric dipoles and a line of magnetic dipoles (a long solenoid) both produce experimentally observed phase shifts similar to that produced by introducing a rectangular block of glass behind one slit of a double-slit interference pattern; the double-slit pattern is shifted while the single-slit envelope remains undisplaced. The quantum explanation for the magnetic interference pattern shift introduced by Aharonov and Bohm in 1959 involves completely different ideas from those suggested by a semiclassical analysis. Experiments planned by Caprez, Barwick, and Batelaan should clarify the connections between classical and quantum theories in connection with the Aharonov-Bohm phase shift.
\end{abstract}




\section{A. Introduction}

The foundations of quantum theory are intimately connected with our understanding of the connections between classical and quantum physics. One of the departures between classical and quantum theories occurred in 1959 when Aharonov and Bohm proposed a new phase shift occurring when charged particles pass around opposite sides of a long solenoid. [1] The experimentally observed Aharonov-Bohm phase shift is said to occur in the absence of classical electromagnetic forces and is said to represent a quantum topological effect which has no classical analogue.

Although the Aharonov-Bohm phase shift is well-verified experimentally, [2] [3] it has been suggested repeatedly during the past thirty-five years that the phase shift may arise not due to "quantum topology" but rather may be due to classical electromagnetic interactions between the passing charges and the solenoid. [4] [5] [6] [7] [8] [9] [10] [11] [12] It is this view which has been sharpened steadily and which very recently has come under experimental investigation. 13] Here we wish to discuss the shifts in particle interference patterns which can arise from electric and magnetic fields.

\section{B. Interference Pattern Shifts}

Particle inference patterns formed when monoenergetic particles pass through two slits to a distant screen are analogous to those obtained when monochromatic light passes through two slits in a wall to a distant screen. In both cases we find a single-slit envelope (associated with the interference of waves passing through a single slit), and then inside the single-slit envelope there is a double-slit interference pattern (associated with the interference of waves passing through two different slits). The interference patterns can be shifted in two distinct arrangements: i) deflection of the entire interference pattern, both the single-slit envelope and the double-slit pattern, or ii) shift of the double-slit pattern leaving the single-slit envelope undisplaced.

i)If a single large prism is place directly behind the two slits so that all light through the slits also passes through the prism, then the interference pattern as a whole is deflected, both the single-slit envelope and the double-slit pattern. An analogous deflection of the particle interference pattern arising from charged particles can be obtained by placing a uniform 
electric field parallel to both the wall with the slits and to the plane formed by the particle paths through the two slits, or else by placing a uniform magnetic field perpendicular to the plane formed by the the charged particle paths through the slits.

ii)If a rectangular piece of glass is placed behind only one of the slits, then the optical interference pattern will suffer a shift in the double-slit interference pattern leaving the single-slit envelope undisplaced. Indeed, if the piece of glass is made sufficiently thick or has a sufficiently large index of refraction so that the waves passing through the glass suffer a spatial delay larger than the coherence length of the light, then the double slit interference pattern will break down while the single slit envelope will remain unchanged. Just such a shift (of the double-slit interference pattern leaving the single-slit envelope undisplaced) can be obtained by placing a line of electric dipoles or a line of magnetic dipoles (a long solenoid) between the charged particle beams passing through the two slits, the line of dipoles oriented perpendicular to the plane formed by the two particle beams. [12]

\section{Shift Due to a Line of Electric Dipoles}

In the electric case, the dipoles are oriented parallel to the wall containing the slits and parallel to the plane formed by the particle beams. The line of dipoles can be obtained by using two line charges $\lambda$ separated by a small distance $2 \varepsilon$ giving a dipole moment per unit length $2 \varepsilon \lambda .[5]$ Positive charged particles which pass through one slit are closer to the positive line charge and so are first repelled while approaching and then attracted back on receding, while the charged particles which pass through the other slit are closer to the negative line charge and so are first attracted while approaching and then repelled while receding. Accordingly, the particles through the different slits have different velocities while passing the line of electric dipoles and so have a relative displacement when the particles have passed far beyond the line of dipoles. The electrostatic situation is analogous to the optical situation where (by use of a piece of glass behind one slit) a relative lag is introduced between the waves which pass through the two slits. The electric energy of interaction of a charged particle $q$ with a line of electric dipoles is given by

$$
U=q V\left(\mathbf{r}_{q}\right)
$$


where $V\left(r_{q}\right)$ is the electrostatic potential of the line of electric dipoles evaluated at the position $\mathbf{r}_{q}$ of the passing charged particle. The relative lag $\Delta Y$ between the charges passing on opposite sides of the line of electric dipoles can be calculated either from the electric fields of the line of electric dipoles acting on the trajectories of the charged particles, or by balancing the electrostatic interaction energies against the kinetic energy of the moving charges $m v_{q} \Delta v_{q}=-U$. The relative spatial lag is given by [5]

$$
\Delta Y=\frac{4 \pi q(2 \varepsilon \lambda)}{m v_{q}^{2}}
$$

where $(2 \varepsilon \lambda)$ represents the electric dipole moment per unit length. Associated with this spatial lag is also a relative time lag

$$
\Delta t=\frac{\Delta Y}{v_{q}}=\frac{4 \pi q(2 \varepsilon \lambda)}{m v_{q}^{3}}
$$

The relative phase shift follows semiclassically as

$$
\Delta \phi=\frac{m v_{q} \Delta Y}{\hbar}=\frac{4 \pi q(2 \varepsilon \lambda)}{\hbar v_{q}}
$$

Exactly this same phase shift can be obtained by inserting the electrostatic potential energy expression $q V\left(\mathbf{r}_{q}\right)$ for a line of electric dipoles into the Schroedinger equation and solving by the WKB approximation assuming that the electrostatic interaction energy is small compared to the kinetic energy of the particles. [10] This phase shift was observed experimentally by Matteucci and Pozzi.[14]

\section{Shift Due to a Line of Magnetic Dipoles (a Solenoid)}

In the magnetic case, a long solenoid is placed between the charged particle beams which pass through the slits so that the axis of the solenoid (and so too the magnetic dipoles) are perpendicular to the plane formed by the particle beams. The magnetic energy of interaction of a charged particle $q$ passing a solenoid with constant currents is given by [15]

$$
U=\frac{q}{c} \mathbf{v}_{q} \cdot \mathbf{A}\left(\mathbf{r}_{q}\right)
$$

where $\mathbf{v}_{q}$ is the velocity of the passing charge and $\mathbf{A}\left(\mathbf{r}_{q}\right)$ is the vector potential in the Coulomb gauge of the solenoid evaluated at the position of the passing charge. We can calculate a possible relative lag between the charged particles which pass on opposite sides of 
the solenoid by using the net Lorentz forces which the passing charges place on the solenoid and then assuming Newton's third law for the forces on the particles, or else by assuming that energy conservation for the magnetic energies occurs by changes in the kinetic energies of the passing particles $m v_{q} \Delta v_{q}=-U$. The possilbe relative spatial lag is given by [5]

$$
\Delta Y=\frac{q \Phi}{m v_{q} c}
$$

for particles passing on opposite sides of the long solenoid of flux $\Phi$ and magnetic moment per unit length $\Phi /(4 \pi)$. Associated with this spatial lag is also a relative time lag

$$
\Delta t=\frac{\Delta Y}{v_{q}}=\frac{q \Phi}{m v_{q}^{2} c}
$$

The relative phase shift follows semiclassically as

$$
\Delta \phi=\frac{m v_{q} \Delta Y}{\hbar}=\frac{q \Phi}{c \hbar}
$$

Alternatively, one can obtain this same phase shift by introducing the vector potential $\mathbf{A}\left(\mathbf{r}_{q}\right)$ of the solenoid into the Schroedinger equation and solving using the WKB approximation assuming that the kinetic energy of the particles is large compared to the magnetic interac-

tion energy $(q / c) \mathbf{v}_{q} \cdot \mathbf{A}\left(\mathbf{r}_{q}\right)$. [10] This phase shift in Eq. (8) was predicted by Aharonov and Bohm[1] and first observed experimentally by Chambers. [2]

\section{E. Comparison of the Electric and Magnetic Phase Shifts}

We have emphasized that the phase shifts due to a line of electric dipoles and to a line of magnetic dipoles (a solenoid) have both been observed experimentally, and both take the same form corresponding to a shift in the double-slit interference pattern while leaving the single-slit envelope undisplaced. Thus these shifts look just like the optical shift obtained by placing a rectangular piece of glass behind only one slit of the two-slit interference arrangement. Furthermore, semiclassical arguments involving energy conservation lead to exactly the observed phase shifts. This suggests that both phase shifts may involve classical lag effects arising from classical electromagnetic forces and velocity changes for the passing charged particles.

However, this is not at all the accepted explanation in the physics literature. The electrostatic situation (in those rare instances when it is acknowledge in the literature) is 
indeed accepted as involving classical electric forces and lags analogous to the optical case. However, it is the magnetic case (the Aharonov-Bohm phase shift) which receives virtually all the attention in the literature.[16] It receives this attention precisely because it is supposed to represent a departure from familiar connections with classical physics. The magnetic situation is said to involve a quantum topological effect having no classical analogue. The magnetic phase shift is said to occur in the absence of classical electromagnetic forces on the passing charged particles, and therefore is said to occur without any velocity changes or relative lags for the passing charges. The novelty of the accepted point of view is emphasized by Silverman who regards the Aharonov-Bohm phase shift as one of the new "mysteries" of

quantum theory. [17] Indeed, the quantum topological point of view is so deeply entrenched that the lag point of view[4] was published only with difficulty in the Physical Review in 1973; in more recent years, the Physical Review has rejected all manuscripts giving further information about the magnetic phase shift from a classical perspective.

\section{F. Experimental Implications}

The classical and quantum analyses for the magnetic phase shift involve totally different perspectives and lead to different experimental implications. The velocity changes, longitudinal displacements, and time lags implied by the classical analysis are all different from the quantum topological point of view which involves no forces or velocity changes. Thus all these aspects become available for experimental discrimination between the two analyses. There is also a further distinction between the classical and quantum analyses. According to presently accepted quantum theory, the Aharonov-Bohm phase shift arises from an enclosed magnetic flux with no need to discuss any interaction between the passing charges and the sources of the magnetic flux. In contrast, the classical lag analysis depends crucially upon the details of the interaction between the passing charged particles and the current-carriers of the magnetic flux.[11] The quantum point of view leads to the expectation that the interaction of a passing charge and a solenoid should remain the same no matter what the size of the solenoid and no matter how the solenoid currents are produced. Indeed, this perspective encourages experimentalists to search for a classical lag effect for charged particles passing a macroscopic solenoid. Such an experiment was performed recently by Caprez, Barwick, and Batelaan, and no classical lag was seen.[18] However, from the classical perspective 
involving the detailed interaction of charges, large and small solenoids may not behave the same way; resistance in the wire of the solenoid can make a difference as to whether or not one should expect to observe an energy-conserving force back on the passing charges.

One may ask how two such totally different points of view can lead to the same experimental prediction for the Aharonov-Bohm phase shift. The relative success of the contrasting points of view may reflect a situation analogous to that when a charged particle passes a conductor. As long as the conductor is a good conductor, the forces on a passing charged particle due to image charges will involve only the physical shape of the conductor and not the detailed composition of the conductor. However, if the conductivity is imperfect or if the induced charges correspond to dielectric behavior, then more information regarding the interaction of the passing charge and the material will be required to account for the behavior of a passing charged particle. In a similar vein for the Aharonov-Bohm phase shift, it may well be that when the resistive energy loss of a solenoid is negligible, then all solenoids behave in the same way regarding energy conservation for a passing charged particle. However, when the resistive energy loss is large, then the energy-conserving interaction becomes small, and the interaction of the particle and solenoid becomes quite different. If the frictional forces (solenoid resistance) are large, then we do not expect to find the time lag because the accelerations of the solenoid charges will be small and hence the back forces at the passing charge will be small.

In the experiments of Moellenstedt and Bayh, [3] where the Aharonov-Bohm phase shift is clearly present, the passage times of electrons past the solenoids is of the order of $10^{-13}$ sec (for a $40 \mathrm{keV}$ electron passing 20 microns from the center of the solenoid). This time is not much longer than the collision time $10^{-14}$ sec in the Drude model for conductivity of a metal. Indeed, Jackson[19] gives $\gamma$ as of the order of $10^{13}$ inverse seconds where $-m \gamma v$ is the resistive damping of a particle of mass $m$ and speed $v$. Thus it is possible that the conservation of energy involving magnetic fields holds in the short-time regime where Moellenstedt and Bayh's experiments were performed, yet would not hold for the much longer passage times for the slower electrons passing the much larger solenoid in the experiment of Caprez, Barwick, and Batelaan. 


\section{G. Crucial Experimental Tests}

The crucial test involving time delay corresponds (in a single experiment) to observing the Aharonov-Bohm phase shift and yet not observing the time delay of Eq. (7). Such an observation would rule out the classical lag interpretation of the Aharonov-Bohm phase shift. For the regimes where the Aharonov-Bohm phase shift has been observed, the time delays would be extraordinarily small. Thus for the experiments of Moellenstedt and Bayh, the time delay in Eq. (7) is of the order of $10^{-21}$ sec.

It is the shifts of particle interference patterns which allow the measurement of extraordinarily small quantities. Thus shift of the interference pattern by a large flux seems the best way to realistically test for the absence of a velocity change. According to the currently accepted quantum point of view, the Aharonov-Bohm phase shift is a purely topological shift, and there is no velocity change and hence no spatial lag for charged particles passing on opposite sides of a solenoid. Accordingly, one can increase the flux in a solenoid by an arbitrary amount and never break down the particle interference pattern. On the other hand, in the classical lag point of view, a sufficiently large solenoid flux will cause a relative lag which is larger than the coherence length associated with the charged particles, and at this point the observed particle interference pattern should break down, just as it does in the optical case. Batelaan's group is hoping to achieve appropriate conditions to observe this possible breakdown. [13]

In the classical analysis, the observed Aharonov-Bohm phase shift due to a solenoid (a line of magnetic dipoles) is analogous to the observed Matteucci-Pozzi phase shift[14] due to a line of electric dipoles.[10] This is not at all the view of currently accepted quantum theory, which accepts the idea that the electrostatic phase shift is due to a classical lag but claims that the magnetic phase shift is due to a new quantum mechanical effect having no classical analogue. Thus ideally one would like to see both these phase shifts (electric and magnetic) tested at the same time with the same coherence length for the electron beam. From both classical and quantum analyses of the electrostatic situation, we believe that we know exactly what is going on involving electrostatic forces leading to a relative lag effect producing the Matteucci-Pozzi electrostatic phase shift. If the interference pattern breaks down for large electric dipole magnitude but not for large magnetic dipole magnitude, then (in contradiction to the classical analysis) the mechanisms for these two interference 
pattern shifts are quite different, as is indeed claimed by currently accepted quantum theory. However, if the interference pattern breaks down for both the electric and the magnetic phase shifts, the currently accepted quantum view is in error.

[1] Y. Aharonov and D. Bohm, "Significance of electromagnetic potentials in quantum theory," Phys. Rev. 115, 485-491 (1959).

[2] R. G. Chambers, "A shift of an electron interference pattern by enclosed magnetic flux," Phys. Rev. Lett. 5, 3-5 (1960).

[3] G. Moellenstedt and W. Bayh, "Messung der kontinuierlichen phasenschiebung von Elecktronenwellen in kraftfeldfreien Raum durch das magnetische Vektorpotential einer Luftspule," Naturwissenschaften 49, 81-82 (1962).

[4] T. H. Boyer, "Classical electromagnetic deflections and lag effects associated with quantum interference pattern shifts: considerations related to the Aharonov-Bohm effect," Phys. Rev. D 8, 1679-1693 (1973).

[5] T. H. Boyer, "The Aharonov-Bohm effect as a classical electromagnetic lag effect: an electrostatic analogue and possible experimental test," Il Nuovo Cimento 100, 685-701 (1987).

[6] T. H. Boyer, "Proposed Aharonov-Casher effect: Another example of an Aharonov-Bohm effect arising from a classical lag," Phys. Rev. A 36, 5083-5086 (1987).

[7] T. H. Boyer, "Does the Aharonov-Bohm effect exist?" Found. Phys. 30, 893-905 (2000).

[8] T. H. Boyer, "Classical electromagnetism and the Aharonov-Bohm phase shift," Found. Phys. 30, 907-932 (2000).

[9] T. H. Boyer, "Classical electromagnetic interaction of a point charge and a magnetic moment: Considerations related to the Aharonov-Bohm phase shift," Found. Phys. 32, 1-36 (2002).

[10] T. H. Boyer, "Semiclassical explanation of the Matteucci-Pozzi and Aharonov-Bohm phase shifts," Found. Phys. 32, 41-49 (2002).

[11] T. H. Boyer, "Darwin-Lagrangian analysis for the interaction of a point charge and a magnet: Considerations related to the controversy regarding the Aharonov-Bohm and Aharonov-Casher phase shifts," J. Phys. A:Math. Gen. 39, 3455-3477 (2006)

[12] T. H. Boyer, "Proposed experimental test for the paradoxical forces associated with the Aharonov-Bohm phase shift," Found. Phys. Lett. 19, 491-498 (2006). 
[13] G. Gronniger, Z. Simmons, S. Gilbert, A. Caprez, and H. Batelaan, "The Aharonov-Bohm effect, phase or force," (preprint).

[14] G. Matteucci and G. Pozzi, "New diffraction experiment on the electrostatic Aharonov-Bohm effect," Phys. Rev. Lett. 54, 2469-2470 (1985).

[15] T. H. Boyer, "Classical electromagnetic interaction of a charged particle with a constantcurrent solenoid," Phys. Rev. D 8, 1667-1697 (1973).

[16] See for example, J. J. Sakurai, Modern Quantum Mechanics Revised Edition (Addison-Wesley, Reading, MA 1994).

[17] M. P. Silverman, More Than One Mystery: Explorations in Quantum Interference (Springer, New York 1995).

[18] A. Caprez, B. Barwick, and H. Batelaan, "A macroscopic test of the Aharonov-Bohm effect," (preprint).

[19] J. D. Jackson, Classical Electrodynamics 3rd ed (Wiley, New York 1999), p. 312. 